\title{
Implementation of Prefabricated Building Systems in Iraq
}

\author{
Zeyad S. M. Khaled
}

\section{Author affiliation: \\ Department of Civil \\ Engineering, College of \\ Engineering, Al-Nahrain \\ University, Baghdad-Iraq. \\ zeyadsmkhalid@gmail.com}

\section{Paper History:}

Received: $23^{\text {rd }}$ July 2020

Revised: $14^{\text {th }}$ Aug. 2020

Accepted: $25^{\text {th }}$ Sep. 2020

\begin{abstract}
In recent decades, Iraq has witnessed several military operations. This has led to huge damages to the infrastructure of some main cities. The traditional construction methods seem not to be able to fulfill the rapid reconstruction works needed, while prefabricated building systems seem to be promising. This paper aims at evaluating the possibilities of using prefabrication systems for schools building as a pilot study for wider adoption. An extensive literature review was carried out to identify the features of construction prefabrication and its requirements. Then a thorough investigation of the possibilities of adopting this approach in Iraq and the major expected obstacles was carried out. A questionnaire survey has been conducted with (96) stakeholders who have experience in prefabricated building projects. The results showed that the highest rating of benefits went to time and productivity, while the highest rating of obstacles went to lack of government support. Therefore the top proposed action included the adoption of a clear governmental strategy for change. Finally, the respondents agreed that school building is the most suitable type of projects to start with.
\end{abstract}

Keywords: Offsite Manufacturing, Industrialized Building System, Construction Module System, Modern Method of Construction.

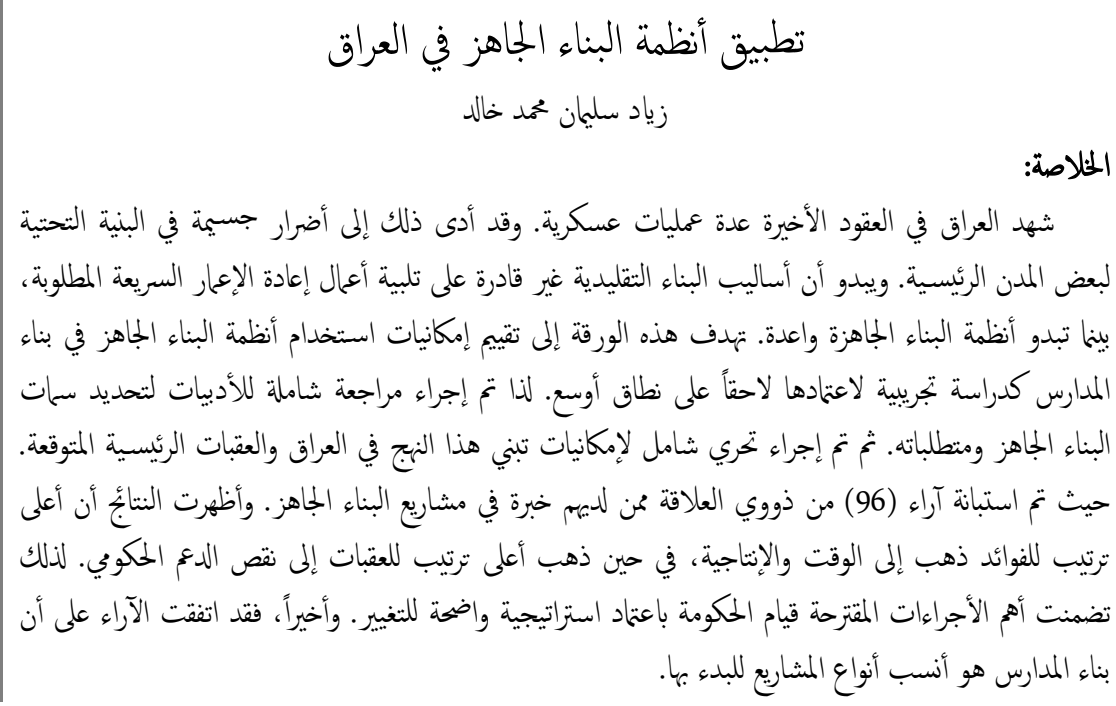

\section{Introduction}

Construction prefabrication is an integrated design, manufacturing and construction process [1], based on mass-production of components manufactured in separate factory and then transported and assembled into a structure with minimal on-site work [2]. It is an ideal approach for certain cases like repetitive elements, speedy execution, dense framing and remote or constricted build locations [3]. The principle is that all repetitive parts of any construction which are difficult, time consuming, costly and/or labour-intensive when constructed on-site can be prefabricated at a plant and then brought and assembled on-site [4]. Furthermore, they can be standardized so that they can be brought from any prefabrication plant meanwhile any plant can supply any prefabrication costumer [5].

Prefabricated concrete elements, steel frames, panels and pods are all included in the general concept of construction prefabrication. A novel approach to construction prefabrication is the modular prefabricated building system, which comprises typically manufactured load-bearing structural components with complete architectural finishes and services. A new initiative is to adopt an

NJES is an open access Journal with ISSN 2521-9154 and eISSN 2521-9162

This work is licensed under a Creative Commons Attribution-NonCommercial 4.0 International License 
open source building concept in which the units can be internationally standardized providing more flexibility, variety and quality of products [6].

The concept of construction prefabrication has not been paid enough attention in Iraq. The Iraqi construction industry failed to utilize modern prefabrication technologies to uplift performance. Traditional construction methods prevailing in Iraq confronted; long duration, high cost, low quality, poor site conditions and considerable material wastage [7 and 8]. Hence, a strategy for employing modern construction approaches has to be adopted. The standardized repetitive nature of construction prefabrication pushes school buildings projects to the front in conducting a pilot study for reconstruction works in Iraq. Nevertheless this study can be a reference for studying such practice in similar countries.

\subsection{Aim and Objectives}

The aim of this research is to investigate the possibilities of adopting prefabricated building systems in Iraq, especially for schools building as a start. This start could aid in countering the bad perception on current precast building and to facilitate the implementation of prefabricated building systems. The following objectives were set:

1. To identify the benefits of adopting construction prefabrication.

2. To investigate the obstacles against adopting construction prefabrication.

3. To determine the readiness to adopt construction prefabrication.

4. To explore actions to facilitate utilizing construction prefabrication.

\subsection{Scope and Limitations}

It seems to be hard for conventional contractors to alter to construction prefabrication because they are not sufficiently equipped with proper technical, managerial and financial capabilities. Furthermore, prefabrication causes reduction in on-site traditional trade works [9]. Hence this research was directed to some major contracting companies especially at the Public Sector for they may have higher capabilities. School buildings were selected to conduct a pilot study because they acquire the highest share of prefabricated projects in Iraq. Moreover, school building projects that were finished in the few past years can provide data up to date. It also should be noted that the ever most of the clients of school buildings in Iraq are governmental agencies.

\subsection{Justification}

Iraq has witnessed several types of military actions in the last few decades. This has led to huge damages to the infrastructure of some main cities. Rapid reconstruction of housing, hospitals, schools and infrastructure utilities is number one objective. Thus, nonconventional construction methods are required for quick and efficient relief. On top of that, it seems that traditional approaches in schools building cannot meet the increasing demand in Iraq, while no local studies are available on the advantages and disadvantages of construction prefabrication. Thus, this study comes to investigate the possibilities of implementing construction prefabrication in schools building in Iraq in an attempt to accelerate filling the huge gap caused by military actions and accumulated shortage as well. The study is a first step to find out leverage opportunities to improve the current practice of reconstruction in Iraq in a wider scale. Another contribution of this research is to improve awareness, increase knowledge and pave the way for prefabrication in similar countries too.

\subsection{Methodology}

This research is an exploratory study to understand current practice and to find out the possibilities of adopting prefabricated building systems and the actions needed. Quantitative and qualitative approaches were used. Special attention was paid to the Projects of the Central Management of Prefabricated School Buildings at the Ministry of Education, for it represents the main client of schools building in Iraq. The staff possesses enough expertise in both conventional and prefabricated building methods. A wide range of data at this Central Management was reviewed. Besides, a questionnaire survey was directed to Iraqi professionals including clients, contractors, manufacturers, consultants, academics and professional association board members. The questionnaire was designed based on information gathered from relevant literature and pilot interviews conducted with some executives among the aforementioned stakeholders in order to address critical issues. Statistical analysis was carried out to verify the findings.

\section{Literature Review}

A comprehensive literature review has been conducted to explore the characteristics and benefits of construction prefabrication and to identify critical success factors.

\subsection{Project Delivery Method}

Shifting to prefabrication is not easy, for it needs to change the philosophy of the construction industry by integrating three major pillars; design, manufacturing and construction. Taking into account that each of these pillars involves many people, techno-logy and processes in which supply chain, marketing and transportation are inherent [10]. Specific features of the products should also be considered for they should be specified, characterized, repeated and optimized. Therefore, the traditional sequential design/bid/build approach is not suitable in which each party acts in relative isolation through a linear process. A collaborative approach, like the bid/ design/build, is fairly suitable for it provides design and construction overlap in which the consultant and contractor coordinate with each other. Meanwhile, an integrated project delivery approach is an ideal one because the manufacturer is also involved in the process [3 and 11]. Figure (1) shows a simplified schematic presentation of project stages in both traditional and prefabricated methods. 


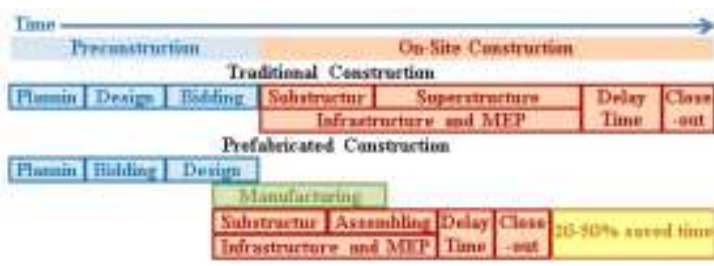

Figure (1): Simplified Presentation of Project Stages

\subsection{Prefabricated Building Systems}

Prefabricated building systems can be classified from least prefabricated systems to more sophisticated ones as: Formwork; Sub-assemblies; Framework; Panelized; Pods; Modular and Hybrid systems [12, 13, 14, 15, 16 and 17]. Regardless of prefabricated formwork which is the lowest rank of construction prefabrication, the evolution of construction prefabrication went through four stages. Stage one was limited to components manufacturing and sub-assembly. Stage two consisted nonvolumetric pre-assembled units which do not enclose usable space. Stage three consisted volumetric preassembled units which enclose fully finished usable space but do not form the building structure. Stage four consisted modular pre-assembled volumetric units which form the actual structure [18].

Recently, an emerging approach for products standardization is introduced by shifting from a closed system where components are made as specifically needed, to an open one where all basic components are standardized offering diversity and flexibility [19]. It is a coordinated unified system for dimensioning spaces, components and fittings so that all elements fit together without cutting or extending even when manufactured by different suppliers [20]. Moreover, industrialization of construction prefabrication went through five distinct levels namely: Prefabrication, Mechanization, Automation, Robotics and Reproduction [1, 4 and 21].

\subsection{Benefits of Construction Prefabrication}

The benefits of employing construction prefabrication can be summarized under the following six headings [22, 23 and 24]:

1. Earlier completion time with higher certainty: The process of prefabrication at centralized plants takes place simultaneously with site preparation providing earlier site assembling. This overlapping plus the ease of erection with minimum formworks at site can speed up construction as much as (50\%) with higher time predictability [25]. Applying the constructability concept might also save time of construction [18].

2. Cost saving with greater certainty and higher profitability: Using mass-production in controlled environment lowers manufacturing outlays due to better controlled processes and economy of scale [1]. Repetitive use and non-labor-intensive works, periodic maintenance, less material waste, logistics and overheads lower on-site outlays [26]. On-site labor can be reduced up to $(50 \%)$ [27]. Saving in foundation sizes and cost is expected due to lower weight of prefabricated structures up to $(30 \%)$ less than conventional ones [28]. Site management improvement is expected as well, due to adopting supply chain method with minimal storage and traffic [29]. All of that can omit up to $(20 \%)$ of total costs. Applying the constructability concept might save cost too [18]. Above all, the IRR will be higher based on life-cycle analysis with earlier pay-back period despite the high initial investment needed for shifting to prefabrication [25].

3. Better quality with higher reliability: Central plants usually have strict quality assurance programs that can maintain high-quality products with serviceability. Using modern techniques for design, manufacturing and construction provide for extensive quality control, adaptability and flexibility. Employing integrated building services provide for efficient usage of products being affordable, comfortable, eco-friendly and fire, heat and sound proof. Standardization, constructability \& repetitive processes would minimize defects [30 and 31].

4. Higher productivity with better workmanship: Productivity and workmanship are affected by the working conditions, continuous training, improvement programs, effective collaboration, efficient communication and supply chain management. Removing nearly $(80 \%)$ of on-site activities to off-site plants provides less congestion, disruption and workers insufficiency so labor productivity might be increased up to $(76 \%)$ [21 and 27].

5. Controlled environment $\&$ lower construction hazards: Less site materials and less wastage with minimal effects of adverse weather provides better controlled environment. Cleaner and tidier site with minimum labor and activities should reduce health and safety risks. Working in centralized plants using mechanization promotes safe and systematic factory working environment. Modern technology improves performance in terms of quality, waste reduction, occupational safety, cost effectiveness and productivity [4].

6. Enhanced sustainability with lower energy consumption: Less construction and manufacturing waste with less air, water and noise pollution all serve to enhance sustainability. Furthermore, it provides better opportunity to use recycled materials and higher possibility to reuse completely prefabricated elements. Modular buildings can be dismantled, refurbished and moved to another location for new use. This also reduces demand for raw materials and minimizes the amount of water and energy needed to create a new building with less $\mathrm{CO}_{2}$ emissions. The operational air-condition energy is considerably reduced due to highly insulating and air-tight modular products. Innovative smart building is much more efficient for it employs building energy management system [14, 32, 33 and 34].

\subsection{Adoption Obstacles}

Perhaps the major obstacle encountered is the bad impression by the people due to many problems encountered old prefabricated buildings such as: leakage from joints, inaccurate connections, poor architectural design and inability to make changes. Therefore, it is also not preferable by designers [21]. The main difficulty in adopting construction 
prefabrication is the high capital needed to start the business due to lack of local technology and facilities for production, testing and erection [35]. Prefabrication needs heavily equipped manufacturing facilities with large area for products storage and equipment movement. It also needs properly skilled staff at both factory and construction sites [6]. Other difficulties are due to its complex interfacing, high technology and transportation requirements which necessitate design and production integration, strict quality management and proper procurement system [36]. The fragmented and project-based nature of the industry, ill-demand, weak awareness, poor knowledge, shortage in relevant education and research also have adverse effects [37]. Lack of erection technical guidelines, codes, standards, regulations and certification make it more difficult to shift [38]. A technical difficulty is the need of additional design requirements concerning products lifting, transporting, placing and fixing in addition to connections and jointing requirements [39]. Above all, the absence of government initiatives, promotion or incentives has a decisive influence [40, 41 and 42].

\subsection{Change Opportunities}

Change opportunities can be assessed based on specific readiness factors which in turn depend on the availability of resources and the ability to utilize them using the required technologies and skills. This can provide for specifying the degree of change needed and the critical areas to focus on [40 and 43].

In order to assure shifting to construction prefabrication, some critical success factors need to be assessed on two levels. The first level is on national scale including: the government strategy and commitment, demand and market conditions, technology transfer, awareness and knowledge, expertise and skills, design and process standardization, information and communication technology, research and development endeavors and coordination and collaboration between parties [20 and 43]. The second level pertain the situation inside the candidate firm including: business and finance, facilities and equipment, design and production integration, constructability and life cycle engineering, organization and leadership style, planning and control techniques, procurement and contracting strategy, supply and storage administration, cost and risk management, transportation and logistics arrangements, productive work environment and quality assurance [36, 40, 43, 44 and 45].

\section{Field Study}

In order to have an outlook on the current practice in school building, follow-up reports of (1427) school buildings projects encompassed by the Central Management of Prefabricated School Buildings at the Ministry of Education were approached. This Central Management is the main client of prefabricated school building in Iraq. These projects were launched in intervals since 2011 to serve all Provinces in Iraq except Kurdistan Region. Unfortunately, in spite of using a computerized follow-up system the documentation process seems to be poor. It was found that only (528) projects were completed till the end of 2019. On-going ones are (899) with a completion rate of $(37 \%)$ on average. Only $(30 \%)$ of these projects were delayed less than $(25 \%)$ of their planned durations while $(70 \%)$ were delayed more. All projects had received an advanced payment ranging from $(10 \%)$ for Private Sector companies up to $(60 \%)$ for Public Sector companies. About $(70 \%)$ of these projects had witnessed cost overrun up to $(20 \%)$ of the contract value. The rest had witnessed much more. The defected work items in these projects ranged from $(5 \%)$ of the items of the Bill of Quantities in $(40 \%)$ of the projects, to $(25 \%)$ of the items of the Bill of Quantities in $(10 \%)$ of them while the others ranged in-between.

Incomplete follow-up data had necessitated conducting a questionnaire survey. Preliminary interviews were held with professionals who are interested in prefabricated projects in order to refine the information gained from the literature review as a step forward to lay out a purposeful questionnaire.

\subsection{Questionnaire Survey}

A questionnaire was directed to professionals at Public and Private Entities including the aforementioned Central Management. Other state organizations and companies approached were subsidiaries of the Ministries of Higher Education, Industry, Transport, Construction, Housing and Municipalities in addition to Engineering Consultancy Bureaus and the Iraqi Engineers Syndicate. A set of questions were asked about construction prefabrication potential benefits, adoption obstacles, readiness for change and actions needed to shift. The benefits were pivoted around; time, cost, quality, productivity, safety and sustainability. The obstacles were pivoted around; initial and additional costs, technology and standardization, awareness and knowledge, expertise and skills, transportation and logistics, stakeholders' intention and government support [23, 46, 47, 48 and 49].

For the sake of specifying stakeholders' responsibilities against required change actions, the readiness factors were reorganized as in Table (1).

A total sum of (96) copies were received out of (120) copies delivered. Likert's (1-5) quintuple scale was provided to the respondents to express the importance of each item in five levels which are (from the lowest to the highest); trivial, low, medium, high and vital effect. The respondents were classified into three groups according to their role, and then a comparison was made between the groups' responses. Group (A) included clients, group (B) included consultants, academics and professional association board members and group (C) included contractors and manufacturers.

\section{The Questionnaire Results 4.1 Part I: The Study Sample}

Tables (2) and (3) show an overview on the attributes of the respondents and the organizations were they belong.

\subsection{Part II: Prefabrication Adoptability}

This part of the questionnaire is devoted to collect the respondents' opinions on the benefits of construction prefabrication, adoption obstacles, 
readiness success factors and proposed actions. Tables (4-8) show the results of these aspects.

\subsection{Part III: Suggestions and Willingness}

The last part of the questionnaire is devoted to explore the respondents' perceptions and attitudes, in addition to any further comments and views. Tables (9) and (10) show the results of this part.

\subsection{Statistical Analysis}

In order to assess the internal consistency of the questionnaire results, reliability and validity tests were conducted based on Cronbach's alpha and P-value measures using SPSS software V.23 [50]. The consistency test was also determined using on-way ANOVA [38], and the average percentage difference was determined using t-test [27]. According to the statistical tests results shown in Tables (11) and (12), it can be said that the questionnaire results acquire sufficient and satisfactory internal consistency and reliability [51].

Table (1): Stakeholders' responsibilities against required change actions

\begin{tabular}{|c|l|l|}
\hline Heading & \multicolumn{1}{|c|}{ Stakeholder } & \multicolumn{1}{c|}{ Readiness Factors } \\
\hline Regulations & Government & $\begin{array}{l}\text { Strategy and commitment, Demand and market conditions, and Technology } \\
\text { transfer. }\end{array}$ \\
\hline $\begin{array}{c}\text { Human } \\
\text { Resources }\end{array}$ & $\begin{array}{l}\text { Government, Universities } \\
\text { and Professional Associations }\end{array}$ & $\begin{array}{l}\text { Government, Manufacturers, } \\
\text { Awareness and knowledge, and Expertise and skills. }\end{array}$ \\
\hline $\begin{array}{l}\text { Technoloctors, Consultants, } \\
\text { Universities and Professional } \\
\text { Associations }\end{array}$ & $\begin{array}{l}\text { Facilities and infrastructure, Design and process standardization, Transportation and } \\
\text { logistics, Information and communication technology, and Research and } \\
\text { development. }\end{array}$ \\
\hline Engineering & $\begin{array}{l}\text { Manufacturers, Contractors } \\
\text { and Consultants }\end{array}$ & $\begin{array}{l}\text { Design, Manufacturing and Construction Integration, Coordination and } \\
\text { collaboration, and Constructability and life cycle engineering. }\end{array}$ \\
\hline Management & $\begin{array}{l}\text { Manufiness and finance, Facilities and equipment, Organization and leadership, } \\
\text { Contranning and control, Procurement and contracting strategy, Supply and storage } \\
\text { Conanagement, Cost \& risk management, and Quality assurance \& work environment. }\end{array}$ \\
\hline
\end{tabular}

Table (2): General Information on the Study Sample

\begin{tabular}{|c|c|c|c|c|c|c|c|c|}
\hline \multirow{2}{*}{$\begin{array}{c}\text { Organization } \\
\text { Sector }\end{array}$} & \multicolumn{4}{|c|}{ Public } & \multicolumn{4}{|c|}{ Private } \\
\hline & \multicolumn{4}{|c|}{76} & \multicolumn{4}{|c|}{20} \\
\hline \multirow{2}{*}{$\begin{array}{l}\text { Organization } \\
\text { Field }\end{array}$} & \multicolumn{4}{|c|}{ Building } & \multicolumn{4}{|c|}{ Others } \\
\hline & \multicolumn{4}{|c|}{84} & \multicolumn{4}{|c|}{12} \\
\hline \multirow{2}{*}{$\begin{array}{c}\text { Organization } \\
\text { Business }\end{array}$} & Client & Contractor & \multicolumn{2}{|c|}{ Manufacturer } & \multicolumn{2}{|c|}{ Consultant } & Academic & Association \\
\hline & 24 & 36 & \multicolumn{2}{|c|}{6} & \multicolumn{2}{|c|}{18} & 6 & 6 \\
\hline \multirow{2}{*}{$\begin{array}{c}\text { Contractors } \\
\text { Class } \\
\end{array}$} & \multicolumn{2}{|c|}{ Civil - first class } & \multicolumn{4}{|c|}{ MEP - first class } & \multicolumn{2}{|c|}{ N/A (not applicable) } \\
\hline & \multicolumn{3}{|c|}{42} & \multicolumn{3}{|c|}{42} & \multicolumn{2}{|c|}{54} \\
\hline \multirow{2}{*}{$\begin{array}{l}\text { Respondent } \\
\text { Education }\end{array}$} & \multicolumn{2}{|l|}{$\mathrm{PhD}$} & \multicolumn{2}{|c|}{ MSc } & \multicolumn{3}{|c|}{$\mathrm{BSc}$} & Diploma \\
\hline & \multicolumn{2}{|l|}{19} & \multicolumn{2}{|c|}{18} & \multicolumn{3}{|c|}{57} & 2 \\
\hline \multirow{2}{*}{$\begin{array}{c}\text { Respondent } \\
\text { Post }\end{array}$} & Top Mgmt. & Middle & Igmt. & Site 1 & gmt. & & upportive & $\mathrm{N} / \mathrm{A}$ \\
\hline & 16 & 4 & & & & & 8 & 6 \\
\hline Respondent & Civil & Mecha & ical & Elec & rical & & Irchitect & Others \\
\hline Job & 58 & 10 & & 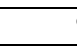 & & & 17 & 2 \\
\hline His Total & $(<6)$ & $(6-$ & & $(11$. & & & $16-20)$ & $(>20)$ years \\
\hline Experience & 0 & 5 & & 2 & & & 28 & 38 \\
\hline His Prefab & Nil & $(<$ & & & & & $(7-9)$ & $(>9)$ years \\
\hline Experience & 0 & 18 & & & & & 19 & 15 \\
\hline
\end{tabular}

Table (3): Previous practice in prefabricated building projects

\begin{tabular}{|c|c|c|c|c|c|c|c|c|c|c|c|c|}
\hline \multirow{2}{*}{$\begin{array}{l}\text { Prefab } \\
\text { systems }\end{array}$} & \multicolumn{3}{|c|}{ Clients } & \multicolumn{3}{|c|}{ Consultants ... } & \multicolumn{3}{|c|}{ Contractors .. } & \multicolumn{3}{|c|}{ All together } \\
\hline & Mean & RII & Rank & Mean & RII & Rank & Mean & RII & Rank & Mean & RII & Rank \\
\hline Formwork & 4.33 & 0.87 & 1 & 2.63 & 0.53 & 1 & 4.12 & 0.82 & 1 & 3.71 & 0.74 & 1 \\
\hline Sub-assembly & 3.63 & 0.73 & 2 & 2.10 & 0.42 & 4 & 3.02 & 0.60 & 4 & 2.89 & 0.58 & 4 \\
\hline Framework & 3.21 & 0.64 & 4 & 2.63 & 0.53 & 2 & 3.26 & 0.65 & 3 & 3.05 & 0.61 & 3 \\
\hline Panelized & 3.33 & 0.67 & 3 & 2.33 & 0.47 & 3 & 3.69 & 0.74 & 2 & 3.18 & 0.64 & 2 \\
\hline Pods & 1.83 & 0.37 & 7 & 1.00 & 0.20 & 7 & 2.12 & 0.42 & 7 & 1.70 & 0.34 & 7 \\
\hline Modular & 1.96 & 0.39 & 6 & 1.00 & 0.20 & 6 & 2.14 & 0.43 & 6 & 1.74 & 0.35 & 6 \\
\hline Hybrid & 2.08 & 0.42 & 5 & 1.00 & 0.20 & 5 & 2.43 & 0.49 & 5 & 1.90 & 0.38 & 5 \\
\hline
\end{tabular}

Table (4): Construction prefabrication potential benefits

\begin{tabular}{|c|c|c|c|c|c|c|c|c|c|c|c|c|}
\hline \multirow{2}{*}{ Benefits } & \multicolumn{3}{|c|}{ Clients } & \multicolumn{3}{|c|}{ Consultants ... } & \multicolumn{3}{|c|}{ Contractors $\ldots$} & \multicolumn{3}{|c|}{ All together } \\
\hline & Mean & RII & Rank & Mean & RII & Rank & Mean & RII & Rank & Mean & RII & Rank \\
\hline Time & 4.18 & 0.84 & 1 & 4.13 & 0.83 & 2 & 3.93 & 0.79 & 1 & 4.38 & 0.88 & 1 \\
\hline Cost & 3.71 & 0.74 & 5 & 3.04 & 0.61 & 3 & 3.87 & 0.77 & 5 & 3.98 & 0.80 & 5 \\
\hline Quality & 3.84 & 0.77 & 3 & 3.58 & 0.72 & 4 & 3.70 & 0.74 & 2 & 4.10 & 0.82 & 2 \\
\hline Productivity & 4.01 & 0.80 & 2 & 3.79 & 0.76 & 1 & 4.10 & 0.82 & 3 & 4.07 & 0.81 & 3 \\
\hline Enclosure & 3.76 & 0.75 & 4 & 3.38 & 0.68 & 5 & 3.67 & 0.73 & 4 & 4.05 & 0.81 & 4 \\
\hline Sustainability & 3.60 & 0.72 & 6 & 3.04 & 0.61 & 6 & 3.53 & 0.71 & 6 & 3.98 & 0.80 & 6 \\
\hline
\end{tabular}


Table (5): Construction prefabrication adoption obstacles

\begin{tabular}{|c|c|c|c|c|c|c|c|c|c|c|c|c|}
\hline \multirow{2}{*}{ Obstacles } & \multicolumn{3}{|c|}{ Clients } & \multicolumn{3}{|c|}{ Consultants ... } & \multicolumn{3}{|c|}{ Contractors $\ldots$} & \multicolumn{3}{|c|}{ All together } \\
\hline & Mean & RII & Rank & Mean & RII & Rank & Mean & RII & Rank & Mean & RII & Rank \\
\hline Investment & 3.50 & 0.70 & 6 & 3.60 & 0.72 & 5 & 3.26 & 0.65 & 5 & 3.43 & 0.69 & 6 \\
\hline Technology & 3.38 & 0.68 & 7 & 3.90 & 0.78 & 2 & 3.19 & 0.64 & 6 & 3.46 & 0.69 & 5 \\
\hline Awareness & 3.58 & 0.72 & 4 & 3.90 & 0.78 & 1 & 3.64 & 0.73 & 3 & 3.71 & 0.74 & 2 \\
\hline Skills & 3.54 & 0.71 & 5 & 3.70 & 0.74 & 3 & 3.40 & 0.68 & 4 & 3.53 & 0.71 & 4 \\
\hline Transport & 3.58 & 0.72 & 3 & 3.40 & 0.68 & 7 & 3.19 & 0.64 & 7 & 3.35 & 0.67 & 7 \\
\hline Stakeholders & 3.67 & 0.73 & 2 & 3.40 & 0.68 & 6 & 3.81 & 0.76 & 2 & 3.65 & 0.73 & 3 \\
\hline Government & 3.71 & 0.74 & 1 & 3.67 & 0.73 & 4 & 4.14 & 0.83 & 1 & 3.89 & 0.78 & 1 \\
\hline
\end{tabular}

Table (6): Construction prefabrication readiness on national scale

\begin{tabular}{|c|c|c|c|c|c|c|c|c|c|c|c|c|}
\hline \multirow{2}{*}{$\begin{array}{c}\text { National } \\
\text { Readiness }\end{array}$} & \multicolumn{4}{|c|}{ Clients } & \multicolumn{3}{c|}{ Consultants ... } & \multicolumn{3}{c|}{ Contractors ... } & \multicolumn{3}{c|}{ All together } \\
\hline Mean & RII & Rank & Mean & RII & Rank & Mean & RII & Rank & Mean & RII & Rank \\
\hline Support & 3.67 & 0.73 & 2 & 4.27 & 0.85 & 2 & 4.19 & 0.84 & 1 & 4.08 & 0.82 & 1 \\
\hline Demand & 3.33 & 0.67 & 7 & 4.10 & 0.82 & 5 & 3.90 & 0.78 & 5 & 3.82 & 0.76 & 5 \\
\hline Equipment & 3.63 & 0.73 & 4 & 4.30 & 0.86 & 1 & 3.55 & 0.71 & 7 & 3.80 & 0.76 & 6 \\
\hline Knowledge & 3.58 & 0.72 & 5 & 4.03 & 0.81 & 6 & 3.95 & 0.79 & 4 & 3.89 & 0.78 & 4 \\
\hline Expertise & 3.92 & 0.78 & 1 & 4.13 & 0.83 & 3 & 4.10 & 0.82 & 2 & 4.06 & 0.81 & 2 \\
\hline Standardizing & 3.67 & 0.73 & 3 & 4.13 & 0.83 & 4 & 4.02 & 0.80 & 3 & 3.97 & 0.79 & 3 \\
\hline ICT & 3.21 & 0.64 & 9 & 3.87 & 0.77 & 9 & 3.48 & 0.70 & 9 & 3.53 & 0.71 & 9 \\
\hline Research & 3.21 & 0.64 & 8 & 3.93 & 0.79 & 8 & 3.52 & 0.70 & 8 & 3.57 & 0.71 & 8 \\
\hline Coordination & 3.46 & 0.69 & 6 & 3.93 & 0.79 & 7 & 3.76 & 0.75 & 6 & 3.74 & 0.75 & 7 \\
\hline
\end{tabular}

Table (7): Construction prefabrication readiness on company scale

\begin{tabular}{|c|c|c|c|c|c|c|c|c|c|c|c|c|}
\hline $\begin{array}{c}\text { Company } \\
\text { Readiness }\end{array}$ & \multicolumn{4}{|c|}{ Clients } & \multicolumn{3}{c|}{ Consultants ... } & \multicolumn{3}{c|}{ Contractors ... } & \multicolumn{3}{c|}{ All together } \\
\hline Finance & 3.50 & 0.70 & 9 & 4.20 & 0.84 & 3 & 3.93 & 0.79 & 2 & 3.91 & 0.78 & 4 \\
\hline Facilities & 3.75 & 0.75 & 4 & 4.53 & 0.91 & 2 & 3.90 & 0.78 & 3 & 4.06 & 0.81 & 2 \\
\hline Design & 3.83 & 0.77 & 2 & 4.60 & 0.92 & 1 & 3.88 & 0.78 & 4 & 4.09 & 0.82 & 1 \\
\hline Constructability & 3.79 & 0.76 & 3 & 4.07 & 0.81 & 4 & 3.62 & 0.72 & 7 & 3.80 & 0.76 & 5 \\
\hline Directing & 3.67 & 0.73 & 5 & 3.87 & 0.77 & 7 & 3.64 & 0.73 & 6 & 3.72 & 0.74 & 6 \\
\hline Planning & 3.63 & 0.73 & 6 & 4.00 & 0.80 & 5 & 3.55 & 0.71 & 8 & 3.71 & 0.74 & 7 \\
\hline Contracts & 3.50 & 0.70 & 10 & 3.67 & 0.73 & 11 & 3.69 & 0.74 & 5 & 3.64 & 0.73 & 8 \\
\hline Supply & 3.54 & 0.71 & 8 & 3.90 & 0.78 & 6 & 3.29 & 0.66 & 11 & 3.54 & 0.71 & 10 \\
\hline Risk & 3.63 & 0.73 & 7 & 3.83 & 0.77 & 9 & 3.48 & 0.70 & 9 & 3.63 & 0.73 & 9 \\
\hline Logistics & 3.17 & 0.63 & 11 & 3.77 & 0.75 & 10 & 3.36 & 0.67 & 10 & 3.44 & 0.69 & 11 \\
\hline Craftsmanship & 3.83 & 0.77 & 1 & 3.83 & 0.77 & 8 & 4.02 & 0.80 & 1 & 3.92 & 0.78 & 3 \\
\hline
\end{tabular}

Table (8): Construction prefabrication proposed actions

\begin{tabular}{|c|c|c|c|c|c|c|c|c|c|c|c|c|}
\hline Actions & \multicolumn{4}{|c|}{ Clients } & \multicolumn{3}{c|}{ Consultants ... } & \multicolumn{3}{c|}{ Contractors ... } & \multicolumn{3}{c|}{ All together } \\
\hline Knowledge & Mean & RII & Rank & Mean & RII & Rank & Mean & RII & Rank & Mean & RII & Rank \\
\hline Academic & 3.96 & 0.79 & 3 & 4.17 & 0.83 & 5 & 3.71 & 0.74 & 5 & 3.92 & 0.78 & 5 \\
\hline Consultant DP & 3.79 & 0.76 & 5 & 4.40 & 0.88 & 3 & 3.83 & 0.77 & 4 & 4.00 & 0.80 & 4 \\
\hline Contractor DP & 4.13 & 0.83 & 1 & 4.40 & 0.88 & 1 & 4.07 & 0.81 & 3 & 4.19 & 0.84 & 2 \\
\hline Labour TP & 4.00 & 0.80 & 2 & 4.40 & 0.88 & 2 & 4.29 & 0.86 & 2 & 4.25 & 0.85 & 1 \\
\hline Codes & 3.92 & 0.78 & 4 & 4.23 & 0.85 & 4 & 4.31 & 0.86 & 1 & 4.19 & 0.84 & 3 \\
\hline Finance & Mean & RII & Rank & Mean & RII & Rank & Mean & RII & Rank & Mean & RII & Rank \\
\hline Costumers & 3.75 & 0.75 & 2 & 4.20 & 0.84 & 1 & 4.07 & 0.81 & 3 & 4.03 & 0.81 & 2 \\
\hline Loans & 3.42 & 0.68 & 5 & 3.63 & 0.73 & 5 & 4.07 & 0.81 & 4 & 3.77 & 0.75 & 5 \\
\hline Tax & 3.46 & 0.69 & 4 & 3.87 & 0.77 & 3 & 4.05 & 0.81 & 5 & 3.84 & 0.77 & 4 \\
\hline Marketing & 3.58 & 0.72 & 3 & 3.67 & 0.73 & 4 & 4.24 & 0.85 & 2 & 3.90 & 0.78 & 3 \\
\hline Payment & 4.00 & 0.80 & 1 & 3.87 & 0.77 & 2 & 4.33 & 0.87 & 1 & 4.10 & 0.82 & 1 \\
\hline TQM & Mean & RII & Rank & Mean & RII & Rank & Mean & RII & Rank & Mean & RII & Rank \\
\hline
\end{tabular}

Table (8) (Continued): Construction prefabrication proposed actions

\begin{tabular}{|c|c|c|c|c|c|c|c|c|c|c|c|c|}
\hline Actions & \multicolumn{4}{|c|}{ Clients } & \multicolumn{3}{c|}{ Consultants ... } & \multicolumn{3}{c|}{ Contractors ... } & \multicolumn{3}{c|}{ All together } \\
\hline Certification & 3.67 & 0.73 & 4 & 4.07 & 0.81 & 3 & 4.10 & 0.82 & 2 & 3.98 & 0.80 & 3 \\
\hline Integration & 4.08 & 0.82 & 1 & 4.27 & 0.85 & 2 & 4.19 & 0.84 & 1 & 4.19 & 0.84 & 1 \\
\hline Compatibility & 3.96 & 0.79 & 2 & 4.30 & 0.86 & 1 & 4.12 & 0.82 & 3 & 4.14 & 0.83 & 2 \\
\hline Solutions & 3.75 & 0.75 & 3 & 3.53 & 0.71 & 5 & 3.55 & 0.71 & 5 & 3.59 & 0.72 & 5 \\
\hline Environment & 3.54 & 0.71 & 5 & 3.90 & 0.78 & 4 & 3.79 & 0.76 & 4 & 3.76 & 0.75 & 4 \\
\hline Productivity & Mean & RII & Rank & Mean & RII & Rank & Mean & RII & Rank & Mean & RII & Rank \\
\hline Mechanizing & 4.17 & 0.83 & 1 & 4.47 & 0.89 & 1 & 4.40 & 0.88 & 1 & 4.36 & 0.87 & 1 \\
\hline Training & 4.04 & 0.81 & 2 & 4.27 & 0.85 & 2 & 4.21 & 0.84 & 2 & 4.19 & 0.84 & 2 \\
\hline Atmosphere & 3.96 & 0.79 & 3 & 3.93 & 0.79 & 3 & 4.05 & 0.81 & 3 & 3.99 & 0.80 & 3 \\
\hline Safety & 3.67 & 0.73 & 5 & 3.83 & 0.77 & 4 & 3.81 & 0.76 & 4 & 3.78 & 0.76 & 4 \\
\hline
\end{tabular}




\begin{tabular}{|c|c|c|c|c|c|c|c|c|c|c|c|c|}
\hline Communicate & 3.92 & 0.78 & 4 & 3.77 & 0.75 & 5 & 3.71 & 0.74 & 5 & 3.78 & 0.76 & 5 \\
\hline Management & Mean & RII & Rank & Mean & RII & Rank & Mean & RII & Rank & Mean & RII & Rank \\
\hline Strategy & 4.25 & 0.85 & 1 & 4.07 & 0.81 & 3 & 4.38 & 0.88 & 1 & 4.25 & 0.85 & 1 \\
\hline Scheduling & 3.96 & 0.79 & 5 & 3.87 & 0.77 & 5 & 3.95 & 0.79 & 2 & 3.93 & 0.79 & 4 \\
\hline Leadership & 4.25 & 0.85 & 2 & 3.93 & 0.79 & 4 & 3.90 & 0.78 & 3 & 4.00 & 0.80 & 2 \\
\hline Collaboration & 4.00 & 0.80 & 3 & 4.17 & 0.83 & 1 & 3.62 & 0.72 & 5 & 3.89 & 0.78 & 5 \\
\hline SCM & 3.96 & 0.79 & 4 & 4.13 & 0.83 & 2 & 3.81 & 0.76 & 4 & 3.95 & 0.79 & 3 \\
\hline
\end{tabular}

Table (9): The respondents' suggested suitable projects types

\begin{tabular}{|c|c|c|c|c|c|c|c|c|c|c|c|c|}
\hline \multirow{2}{*}{$\begin{array}{c}\text { Candidate } \\
\text { projects type }\end{array}$} & \multicolumn{4}{|c|}{ Clients } & \multicolumn{3}{c|}{ Consultant ... } & \multicolumn{3}{c|}{ Contractors ... } & \multicolumn{3}{c|}{ All together } \\
\hline Mean & RII & Rank & Mean & RII & Rank & Mean & RII & Rank & Mean & RII & Rank \\
\hline Residential & 4.25 & 0.85 & 2 & 4.13 & 0.83 & 2 & 4.36 & 0.87 & 1 & 4.26 & 0.85 & 2 \\
\hline Offices & 3.00 & 0.60 & 3 & 3.63 & 0.73 & 4 & 3.26 & 0.65 & 4 & 3.31 & 0.66 & 3 \\
\hline Health & 2.88 & 0.58 & 4 & 3.50 & 0.70 & 5 & 3.43 & 0.69 & 3 & 3.31 & 0.66 & 4 \\
\hline Educational & 4.38 & 0.88 & 1 & 4.23 & 0.85 & 1 & 4.26 & 0.85 & 2 & 4.28 & 0.86 & 1 \\
\hline Industrial & 2.54 & 0.51 & 7 & 3.93 & 0.79 & 3 & 3.21 & 0.64 & 5 & 3.27 & 0.65 & 5 \\
\hline Tourism & 2.46 & 0.49 & 8 & 3.17 & 0.63 & 8 & 3.19 & 0.64 & 7 & 3.00 & 0.60 & 8 \\
\hline Commercial & 2.71 & 0.54 & 5 & 3.23 & 0.65 & 7 & 3.12 & 0.62 & 8 & 3.05 & 0.61 & 7 \\
\hline Utilities & 2.63 & 0.53 & 6 & 3.43 & 0.69 & 6 & 3.19 & 0.64 & 6 & 3.13 & 0.63 & 6 \\
\hline
\end{tabular}

Table (10): The respondents' acceptance level and willingness

\begin{tabular}{|c|c|c|c|c|c|c|c|c|c|c|c|c|}
\hline \multirow{2}{*}{$\begin{array}{c}\text { Respondents' } \\
\text { Perception }\end{array}$} & \multicolumn{4}{|c|}{ Clients } & \multicolumn{3}{c|}{ Consultants ... } & \multicolumn{3}{c|}{ Contractors ... } & \multicolumn{4}{c|}{ All together } \\
\cline { 2 - 26 } & Mean & RII & Rank & Mean & RII & Rank & Mean & RII & Rank & Mean & RII & Rank \\
\hline Acceptance & 3.21 & 0.64 & Med. & 2.23 & 0.45 & Low & 3.79 & 0.76 & High & 3.16 & 0.63 & Med. \\
\hline willingness & 3.67 & 0.73 & High & 2.67 & 0.53 & Med. & 4.07 & 0.81 & High & 3.53 & 0.71 & High \\
\hline
\end{tabular}

Table (11): Overall statistical test results

\begin{tabular}{|c|c|c|c|c|c|c|c|c|c|}
\hline \multirow[b]{2}{*}{ Group } & \multicolumn{2}{|c|}{ Reliability } & \multicolumn{7}{|c|}{ ANOVA } \\
\hline & $\mathrm{N}$ & $\alpha$ & People & Items & $\begin{array}{l}\text { Sum of } \\
\text { Squares }\end{array}$ & $\mathrm{df}$ & $\begin{array}{l}\text { Mean } \\
\text { Square }\end{array}$ & $\mathrm{F}$ & Sig. \\
\hline \multirow{5}{*}{$\begin{array}{c}\text { Group } \\
\text { A }\end{array}$} & \multirow{5}{*}{75} & \multirow{5}{*}{.934} & \multicolumn{2}{|c|}{ Between People } & 340.284 & 23 & 14.795 & \multirow{5}{*}{6.693} & \multirow{5}{*}{.000} \\
\hline & & & \multirow{3}{*}{$\begin{array}{l}\text { Within } \\
\text { People }\end{array}$} & Between Items & 486.444 & 74 & 6.574 & & \\
\hline & & & & Residual & 1671.716 & 1702 & .982 & & \\
\hline & & & & Total & 2158.160 & 1776 & 1.215 & & \\
\hline & & & & Total & 2498.444 & 1799 & 1.389 & & \\
\hline \multirow{5}{*}{$\begin{array}{c}\text { Group } \\
\text { B }\end{array}$} & \multirow{5}{*}{75} & \multirow{5}{*}{.890} & \multicolumn{2}{|c|}{ Between People } & 211.833 & 29 & 7.305 & \multirow{5}{*}{21.120} & \multirow{5}{*}{.000} \\
\hline & & & \multirow{3}{*}{$\begin{array}{l}\text { Within } \\
\text { People }\end{array}$} & Between Items & 1254.166 & 74 & 16.948 & & \\
\hline & & & & Residual & 1722.100 & 2146 & .802 & & \\
\hline & & & & Total & 2976.267 & 2220 & 1.341 & & \\
\hline & & & & Total & 3188.100 & 2249 & 1.418 & & \\
\hline \multirow{5}{*}{$\begin{array}{l}\text { Group } \\
\text { C }\end{array}$} & \multirow{5}{*}{75} & \multirow{5}{*}{.839} & \multicolumn{2}{|c|}{ Between People } & 202.295 & 41 & 4.934 & \multirow{5}{*}{12.031} & \multirow{5}{*}{.000} \\
\hline & & & \multirow{3}{*}{$\begin{array}{l}\text { Within } \\
\text { People }\end{array}$} & Between Items & 706.923 & 74 & 9.553 & & \\
\hline & & & & Residual & 2409.157 & 3034 & .794 & & \\
\hline & & & & Total & 3116.080 & 3108 & 1.003 & & \\
\hline & & & & Total & 3318.375 & 3149 & 1.054 & & \\
\hline \multirow{5}{*}{$\begin{array}{c}\text { All } \\
\text { Groups }\end{array}$} & \multirow{5}{*}{75} & \multirow{5}{*}{.893} & \multicolumn{2}{|c|}{ Between People } & 798.477 & 95 & 8.405 & \multirow{5}{*}{28.553} & \multirow{5}{*}{.000} \\
\hline & & & \multirow{3}{*}{$\begin{array}{l}\text { Within } \\
\text { People }\end{array}$} & Between Items & 1906.660 & 74 & 25.766 & & \\
\hline & & & & Residual & 6343.846 & 7030 & .902 & & \\
\hline & & & & Total & 8250.507 & 7104 & 1.161 & & \\
\hline & & & & Total & 9048.983 & 7199 & 1.257 & & \\
\hline
\end{tabular}

Table (12): Some independent samples tests results

\begin{tabular}{|c|c|c|c|c|c|c|c|c|c|c|}
\hline \multirow{3}{*}{$\begin{array}{l}\text { Proposed } \\
\text { Actions }\end{array}$} & \multirow{3}{*}{$\begin{array}{c}\text { Equal } \\
\text { variances }\end{array}$} & \multirow{2}{*}{\multicolumn{2}{|c|}{$\begin{array}{l}\text { Levene's } \\
\text { Test for } \\
\text { Equality }\end{array}$}} & \multicolumn{7}{|c|}{ t-test for Equality of Means } \\
\hline & & & & \multirow[b]{2}{*}{$\mathrm{t}$} & \multirow[b]{2}{*}{$\mathrm{df}$} & \multirow{2}{*}{$\begin{array}{c}\text { Sig. } \\
2- \\
\text { tailed }\end{array}$} & \multirow{2}{*}{$\begin{array}{l}\text { Mean } \\
\text { Diff. }\end{array}$} & \multirow{2}{*}{$\begin{array}{l}\text { Std. } \\
\text { Error } \\
\text { Diff. }\end{array}$} & \multicolumn{2}{|c|}{$\begin{array}{l}95 \% \text { Confidence } \\
\text { Interval of Diff. }\end{array}$} \\
\hline & & $\mathrm{F}$ & Sig. & & & & & & Lower & Upper \\
\hline \multirow{2}{*}{ Academic } & assumed & 2.917 & .092 & 1.043 & 64 & .301 & .24405 & .23402 & -.22346 & .71155 \\
\hline & not & & & 1.071 & 51.882 & .289 & .24405 & .22795 & -.21338 & .70148 \\
\hline \multirow{2}{*}{ Consultant } & assumed & .724 & .398 & -207 & 64 & .837 & -.04167 & 20177 & -.44474 & .36141 \\
\hline & not & & & -.202 & 44.564 & .841 & -.04167 & .20676 & -.45822 & .37489 \\
\hline \multirow{2}{*}{ Contractor } & assumed & 3.803 & .056 & .225 & 64 & .823 & .05357 & .23809 & -.42208 & .52922 \\
\hline & not & & & .206 & 37.000 & .838 & .05357 & .25964 & -.47251 & .57965 \\
\hline \multirow{2}{*}{ Labour } & assumed & .002 & .969 & -1.438 & 64 & .155 & -.28571 & .19865 & -.68256 & .11113 \\
\hline & not & & & -1.318 & 36.896 & .196 & -.28571 & .21683 & -.72510 & .15367 \\
\hline \multirow{2}{*}{ Codes } & assumed & .542 & .464 & -1.758 & 64 & .084 & -.39286 & .22346 & -.83927 & .05356 \\
\hline & not & & & -1.710 & 44.155 & .094 & -.39286 & .22969 & -.85572 & .07000 \\
\hline Costumers & assumed & .345 & .559 & -1.377 & 64 & .173 & -.32143 & .23348 & -.78787 & 14501 \\
\hline
\end{tabular}




\begin{tabular}{|c|c|c|c|c|c|c|c|c|c|c|}
\hline & not & & & -1.327 & 42.964 & .191 & -.32143 & .24215 & -.80979 & .16693 \\
\hline \multirow{2}{*}{ Loans } & assumed & .099 & .754 & -3.065 & 64 & .003 & .65476 & .21365 & -1.0815 & -.2279 \\
\hline & not & & & -3.073 & 48.399 & .003 & 65476 & .21307 & -1.0830 & -.2264 \\
\hline \multirow{2}{*}{ Tax } & assumed & 880 & .095 & -2.422 & 64 & .018 & 58929 & .24331 & -1.0753 & -.1032 \\
\hline & not & & & -2.302 & 41.114 & .026 & .58929 & .25603 & -1.1063 & -.0722 \\
\hline \multirow{2}{*}{ Marketing } & assumed & 936 & .337 & 3.345 & 64 & .001 & 65476 & .19575 & -1.0458 & -.2637 \\
\hline & not & & & -3.224 & 42.927 & .002 & 65476 & .20307 & -1.0643 & -.2452 \\
\hline \multirow{2}{*}{ Payment } & assumed & 748 & .004 & -1.662 & 64 & .101 & .33333 & .20060 & .73408 & 06741 \\
\hline & not & & & -1.423 & 30.718 & .165 & -.33333 & .23419 & -.81114 & .14448 \\
\hline \multirow{2}{*}{ Certification } & assumed & 2.27 & .000 & -2.204 & 64 & .031 & -.42857 & .19443 & -.81700 & -.0401 \\
\hline & not & & & -1.868 & 29.898 & .072 & -.42857 & .22944 & -.89721 & .04007 \\
\hline \multirow{2}{*}{ Integration } & assume & 677 & .200 & -.556 & 64 & .580 & .10714 & .19274 & -.49218 & .27789 \\
\hline & & & & -.516 & 38.436 & .608 & .10714 & .20745 & -.52695 & .31267 \\
\hline \multirow{2}{*}{ Compatibility } & & 010 & .921 & -.652 & 64 & .517 & -.16071 & .24644 & -.65303 & 33160 \\
\hline & & & & -.642 & 45.701 & .524 & -.16071 & .25046 & .66496 & 34353 \\
\hline \multirow{2}{*}{ Solutions } & assume & .340 & .562 & .839 & 64 & .405 & .20 & .24128 & -.27964 & .68440 \\
\hline & & & & .821 & 45.007 & .416 & .202 & .24646 & -.29400 & .69877 \\
\hline \multirow{2}{*}{ Environment } & assum & .951 & .167 & -1.029 & 64 & .308 & -.24405 & .23727 & -.71806 & .22996 \\
\hline & not & & & -.965 & 39.614 & .340 & -.24405 & .25280 & -.75513 & .26704 \\
\hline \multirow{2}{*}{ Mechanizing } & assumec & 2.975 & .089 & -1.081 & 64 & .284 & -.23810 & .22033 & -.67826 & .20207 \\
\hline & & & & -.962 & 34.070 & .343 & -.23810 & .24739 & -.74 & .26462 \\
\hline \multirow{2}{*}{ Trainin } & assume & 2.306 & .134 & -.853 & 64 & .397 & & .20237 & & .23166 \\
\hline & & & & -.761 & 34.182 & .452 & 262 & & & .28850 \\
\hline \multirow{2}{*}{ Atmosphere } & assum & .698 & .407 & -.487 & 64 & .628 & -.08929 & .18336 & & .27702 \\
\hline & not & & & -.461 & 40.677 & .647 & -.08929 & .19364 & -.48044 & .30187 \\
\hline \multirow{2}{*}{ Safety } & assumed & 4.643 & .035 & -.609 & 64 & .545 & -.14286 & .23463 & -.61158 & .32587 \\
\hline & not & & & -.561 & 37.474 & .578 & -.14286 & .25474 & -.65 & .37307 \\
\hline \multirow{2}{*}{ Communicate } & assumed & .000 & .995 & .972 & 64 & .335 & .20238 & .20829 & -.21372 & .61848 \\
\hline & & & & .914 & 39.866 & .366 & .20238 & .22144 & -.24522 & .64998 \\
\hline \multirow[t]{2}{*}{ Strategy } & assur & .011 & .915 & -.661 & 64 & .511 & -.13095 & .19822 & -.52694 & .26503 \\
\hline & & & & -.6 & 46.45 & .5 & & & & .27227 \\
\hline \multirow[t]{2}{*}{ Scheduling } & assume & .929 & .339 & .029 & 64 & .977 & .00595 & .20446 & -.40251 & .41442 \\
\hline & not & & & .028 & 43.499 & .978 & .00595 & .21120 & -.41982 & .43173 \\
\hline \multirow[t]{2}{*}{ Leadership } & assumed & .267 & .607 & 1.796 & 64 & .077 & .34524 & .19223 & -.03878 & .72926 \\
\hline & not & & & 1.811 & 49.179 & .076 & .34524 & .19069 & -.03792 & .72840 \\
\hline \multirow[t]{2}{*}{ Collaboration } & assumed & .708 & .403 & 1.797 & 64 & .077 & .38095 & .21194 & -.04244 & .80435 \\
\hline & & & & 1.702 & 40.653 & .096 & .38095 & .22386 & -.07126 & .83317 \\
\hline \multirow[t]{2}{*}{ SCM } & assumed & .303 & .584 & .723 & 64 & .472 & .14881 & .20589 & -.26250 & .56012 \\
\hline & not & & & .722 & 47.861 & .474 & .14881 & .20609 & -.26560 & .56322 \\
\hline
\end{tabular}

\section{Findings and Discussion}

It should be mentioned that the respondents were found to be familiar with construction prefabrication techniques, so there opinions were of great help. Group (A - clients), group (B - contractors and manufacturers) and group (C - consultants, academics and professional association board members) were specifically found to have enough knowledge and experience in formwork, sub-assemblies, framework and panelized systems of construction prefabrication. Meanwhile they lack knowledge and experience in modern methods like pods and modular ones which are not familiar yet in Iraq. On the other hand, they all have enough experience in school buildings works using both conventional and prefabrication methods. Group (B - contractors and manufacturers) believe that they can survive in a modern construction prefabrication market in spite of the challenges.

Considering Table (4), it can be noticed that the highest rating of construction prefabrication benefits went to the speed of construction (time and productivity were confirmed by all three groups) which is the most vital concern rapid reconstruction. The next benefit rating went to better quality control affirmed by better controlled environment. Cost and sustainability came next but they also possess very high importance just like other expected benefits of prefabrication. The inferior rank is attributed to the inconvenient current practice where prefabricated projects suffered from cost overrun as already aforementioned. Furthermore, sustainability aspects are not paid enough attention in Iraq so far.

From Table (5), it can be noticed that the major obstacles confronting the change to prefabrication is lack of governmental support accompanied with lack of stakeholders' awareness and intention. Meanwhile, other obstacles especially the lack of skills and technology possess high importance as well. When crosschecking these findings with the readiness for change on national scale in Iraq, as illustrated in Table (6), it can be noticed that government role comes above-all including providing support, standardization, persistent demand and knowledge /expertise development programs. On the other hand, when looking at the readiness for change on company scale, it can be noticed that the most critical success factors are; design, facilities, craftsmanship, finance and constructability, as illustrated in Table (7). This can be attributed to the vital role of design in attracting customers and maintaining their after-use satisfaction so that the business can survive. Furthermore, well-trained craftsmanship using constructible 
design might insure successful accomplishment of projects within planned time, cost and quality.

In addition, lack of proper modern facilities is the other side of the same coin of finance shortage. The parameters that affect the life-cycle economic analysis of establishing a construction prefabrication business are; initial cost, manufacturing cost, repair and maintenance cost, transportation cost, erection cost, economic life of assets and interest/inflation rates. Saved labour, material and time should also be taken into account. The initial cost should include all expenses associated with labour, material and facilities. Facilities expenses, in turn, should include purchasing, installation, depreciation and investment costs of equipment and its work place.

The bottom line of this study is to reach some feasible actions that should be practical and rational enough to be taken. The proposed actions were classified first under five headings; knowledge, finance, quality, productivity and management. Each heading comprises a set of five actions as listed in Table (8). It was found that the industry is in need of providing modern equipment, materials and tools for manufacturing and assembling. It should also adopt an effective program for contractors' development and labor training to be able to utilize modern technology effectively. Moreover, collaborative endeavor should be fostered by main players (designers, manufacturers and contractors) to provide an integrated design-make-erect process. A clear binding strategy should be promoted to adopt the actions.

Finally, the respondents agree with the suggested approach of starting the implementation of modern prefabrication in the field of school building at first. This might assure a successful start because the respondents considered education projects as the most suitable type of construction projects for prefabrication as shown in Table (9). Residential projects comes next and then office buildings. From Table (10) it can be noticed that the willingness of the industry stakeholders seems to be high enough to launch such a national campaign.

\section{Conclusions}

The Iraqi construction sector is still practicing conventional construction methods that are accused to be extravagant, perilous and scrubby. Realization of the benefits of construction prefabrication among respondents is significant, but the readiness (on national and company's levels) is extremely low and many obstacles are confronted.

According to the findings of this research, the following benefits of adopting modern construction prefabrication can be highlighted;

1. Time-efficiency.

2. Long-term cost benefits.

3. Less skilled workforce on-site.

4. Resources integration.

5. Pre-planned process.

6. High-quality controlled environment.

Since military actions ruin local production facilities, supply arrangements and transportation systems, causing difficulties in timely delivery of resources to on-site works, thus, construction prefabrication can offer a feasible solution for the most vital recovery concerns; time, cost and quality assurance.

On the other hand, low readiness in Iraq can be attributed to the following factors:

1. Lack of research and development programs to facilitate adopting construction prefabrication.

2. Discontinuity of prefabricated projects which led to contradictious opinions among stakeholders.

3. Poor logistics and transportation facilitation make it hard for local construction companies to shift to construction prefabrication.

Furthermore, it can be concluded that the adoption of construction prefabrication in Iraq is confronted by the following obstacles:

1. High capital cost for facilities and storage.

2. Transportation difficulties and additional expenses.

3. Lack of demand and market discontinuity, fragmentation and diversity.

4. Absence of local regulations, codes, specifications and standards.

5. Lack of technology and shortage of expertise engineers and skilled labour.

6. Low awareness accompanied with inadequate relevant academic programs and research.

7. Bad reputation due to several defects in existing buildings, inability to make changes and weakness of connections.

Nevertheless, Public sector companies have better chance to adopt construction prefabrication than private ones, for they have higher capabilities to possess nonconventional construction technologies, sophisticated management systems, and financing.

\section{Recommendations}

As long as the industry focuses on the short term gains, it will be considered unattractive to establish local construction prefabrication industry. This is because the aforementioned obstacles make it uneconomical to the private sector to invest in central production plants. Even though sub-contractors are able to sustain in some on-site traditional trades, main contractors are unable to shift to construction prefabrication without government support.

Based on the findings and conclusions of this research, the following recommended actions are suggested:

1. Government support: Effective government support should, at least, include funding aid, developing market capacity and capability, tax exemption \& levy reduction, leasing for technology transfer, reduction on import duty, improving training, redirecting education, strengthening research, adopting innovation and issuing of relevant legislations, codes and standards. For instance, a flexible bank loan or attractive financial package by the government could encourage main contractors to integrate. Some of them can be directed to join in order to increase their capabilities in convoying with modern technology and installation skills. Others can be directed to switch to manufacturers. 
2. Radical changes in the industry's philosophy: This change should, at least, include changing the conventional mindset on industrialization by changing the scope from project-based to productbased, reorienting design and specification from short-term to long-term concerns, shifting the manual technology used to mechanization and upgrading transportation facilities from traditional to modern. In this sense; the bases of considering gains and profit stability will be changed from single project to mass production, duration concerns will be changed from separate timelines to overlapping, environment concerns will be changed from harsh to friendly activities and the number of unskilled labor needed will be changed from high to low.

3. Adopting a clear governmental strategy: A proper strategy should be set to provide a timely roadmap that outline steps to promote rapid adoption of construction prefabrication in Iraq. Government agencies, Universities, professional associations, consultants, developers, contractors and manufacturers should collaborate to develop a strategy with a top-down vision and commitment. The vision should have broader view of construction prefabrication implementation aiming at providing high level outcomes, efficiency and competency. The mission should aim at providing speed, economy, quality and sustainability.

4. Integration of design, manufacture and construction: Lack of standardized attractive design discourages successful change. Integration between design, manufacturing and constructing activities is pivotal. A key factor is initializing the desire for collaboration between clients, consultants, manufacturers and contractors by adopting joint ventures, partnering or consortium in a 'one-stop center' approaches. This can provide for effective dealing with complex interfacing of design, manufacture, transportation and installation. It might also ensure efficient process sequence providing expertise and practitioners sharing.

5. Establishing a Construction Industry Development Board: Establishing such an entity is essential to guide this endeavor providing certification and standardization for manufacturing and site works, in addition to development programs for training, education, leadership, organization, design integration, information technology, cost control, risk management, processes optimization, procurement and supply chain. This Board could take the lead to conduct studies, forecast demand, publish references, enforce prefabrication by-laws, and develop national standards for skills and unified components designs, quality assurance programs, data base, software, and websites.

\section{References}

[1] S. S. Kamaruddin, M. F. Mohammad and R. Mahbub, "Enhancing the Quality of Life by Adopting IBS: An economic perspective on mechanization and automation", Procedia - Social and Behavioral Sciences, Elsevier, 101 (2013) 71 80, 2013.
[2] W. A. Thanoon, L.W. Peng, M. R. Abdul Kadir, M. S. Jaafar and M. S. Salit, "The Essential Characteristics of Industrialized Building System", Proceedings of International Conference on Industrialized Building Systems, Kuala Lumpur, Malaysia, 10-11 September, 2003, p. 283-292.

[3] James Wilson of Building Green, Inc., "Design for Modular Construction: An Introduction for Architects", A Guide prepared after the American Institute of Architects (AIA) and the National Institute of Building Sciences, USA, 2019.

[4] A. Rashidi and R. Ibrahim, "Industrialized Construction Chronology: The Disputes and Success Factors for a Resilient Construction Industry in Malaysia", the Open Construction and Building Technology Journal, Bentham Open, Vol. 11, 2017, p. 286-300.

[5] M. I.Din, N. Bahri, M. A. Dzulkifly, M. R. Norman, K. M. Kamar and Z. A. Hamid, "The adoption of Industrialized Building System (IBS) Construction in Malaysia: The history, policies, experiences and lesson learned", Proceedings of 29th International Symposium on Automation and Robotics in Construction - ISARC, The International Association for Automation and Robotics in Construction, Eindhoven, The Netherlands, 2012.

[7] Z. S. M. Khaled, R. S. Abid-Ali and M. F. Hasan, "Modeling the Completion Time of Public School Building Projects Using Neural Networks", Civil Engineering Journal, 3(12); 1266-1277, 2017.

[8] Z. S. M. Khaled, Q. J. Frayyeh and G. K. Aswed, "Modeling Final Costs of Iraqi Public School Projects Using Neural Networks", International Journal of Civil Engineering and Technology, 5(7): 42-54, 2014.

[6] Y. Setiani, "Implementation of Industrialized Building System (IBS) in Malaysia Construction Industry", Seminar Nasional Universitas Budi Luhur 14 Agustus 2009, Jakarta, Indonesia.

[9] M. Theong, R. A. Rashid and C. Chai, "Implication of Industrialized Building System (IBS): The Case of Trade Contractors in Malaysian", International Journal of Engineering and Technology, Science Publishing Corporation, 7 (2.29), p. 766-771, 2018.

[10] M. F. Musa, M.R. Yusof, N. S. Samsudin and F. M. Halil, "The Industrialized Building System Modular System (IBSMS) Framework", Proceedings of 5th AMER International Conference on Quality of Life, Bangkok, Thailand, 25-27 February 2017, E-BPJ, 2(5), March 2017 (p.105-113).

[11] M. R. Yusof, M. F. Musa, N. S. Samsudin, M. F. Mohammad and M. N. Baharuddin, "Industrialized Building System Plan of Work", International Review of Management and Marketing, 6(S7), p. 299-305, Special Issue for International Soft Science Conference, 11-13 April 2016, Universiti Utara Malaysia.

[12] M. F. Musa, M. F. Mohammad, M. R. Yusof and R. Mahbub, "The Way Forward for Industrialized Building System (IBS) in Malaysia”, Proceedings of the International Civil and Infrastructure 
Engineering Conference 2014, Springer Singapore, p. 163-175.

[13] Z. Ismail and A. Abdul Rahim, "Adaptability and Modularity in Housing: A Case Study of Raines Court and Next21", International Conference on Universal Design in Built Environment, International Islamic University Malaysia, 2011.

[14] M. F. Musa, M. F. Mohammad, M. R. Yusof and R. Mahbub, "Characteristics of Modular Construction: Meeting the Needs of Sustainability and Innovation", IEEE Colloquium on Humanities, Science and Engineering Research, Malaysia, p. 216-221, 2014.

[15] L. Kyjakova, T. Mandicak and P. Mesaros, "Modern Methods of Constructions and their Components", Journal of Engineering and Architecture, American Research Institute for Policy Development, Vol. 2, No. 1, pp. 27-35, 2014.

[16] NHBC Foundation, "Modern Methods of Construction: Views from the Industry", National House Building Council, IHS BRE Press, England, 2016.

[17] L. Kyjakova and R. Baskova, "Advantages and Disadvantages of Modern Methods of Construction used for Modular Schools in Slovakia", Technical Transactions - Civil Engineering, Cracow University of Technology, Poland, vol. 113, issue 1-B, p. 35-41, 2016.

[18] M. N. A. Azman, M. S. S. Ahamad and W. M. A. Hussin, "Comparative Study on Prefabrication Construction Process", International Surveying Research Journal (ISrJ), Royal Institution of Surveyors Malaysia, Vol. 2 No. 1, p. 45-58, 2012.

[19] M. N. M. Nawi, M. N. A. Azman, N. Baluch, K. A. M. Kamar and D. Z. A. Hamid, "Study on the Use of Industrialized Building System in Malaysian Private Construction Projects", ARPN Journal of Engineering and Applied Sciences, Vol. 10, No. 17, p. 7368-7374, 2015.

[20] K. X. How, "Barriers in the Implementation of Industrialized Building System in Malaysian Construction Industry", MSc Thesis, Faculty of Civil Engineering, University of Technology, Malaysia, 2009.

[21] M. F. Mohammad, A. S. Baharin, M. F. Musa and M. R. Yusof, "The Potential Application of IBS Modular System in the Construction of Housing Scheme in Malaysia", Procedia - Social and Behavioral Sciences, Elsevier, 222 (2016) 75 82.

[22] M. Q. Oliewy, K. N. Mustapha and B. S. Mohammad, "Advantages of industrialized building system in Malaysia", IEEE Student Conference on Research and Development, SCORED 2009, Putra University, Serdang, Malaysia.

[23] I. Jabar, F. Ismail and A. A. Mustafa, "Issues in Managing Construction Phase of IBS Projects", Procedia - Social and Behavioral Sciences, Elsevier, 101 (2013) 81 - 89, 2013

[24] N. Bertram, S. Fuchs, J. Mischke, R. Palter, G. Strube and J. Woetzel, "Capital Projects and Infrastructure: Modular construction: From projects to products", McKinsey and Company, Chicago, USA, 2019.

[25] P. Ghannad, Y-C. Lee and J. O. Choi, "Investigating Stakeholders' Perceptions of Feasibility and Implications of Modular Construction-Based Post-Disaster Reconstruction", Modular and Offsite Construction (MOC) Summit Proceedings, Banff, Alberta, Canada, 504-513, 2019.

[26] M. N. M. Nawi, F. A. Abu Hanifa, K. A. M. Kamar, A. Lee and M. N. A. Azman, "Modern Method of Construction: An Experience from UK Construction Industry", Australian Journal of Basic and Applied Sciences, 8(5) Special 2014, P. 527-532, 2014.

[27] T. A. Majid, M. N. A. Azman, S. A. S. Zakaria, A. S. Yahia, S. S. Zaini, M. S. S. Ahmad and M. H. Hanafi, "Quantitative Analysis on the Level of IBS Acceptance in the Malaysian Construction Industry", Journal of Engineering Science and Technology, School of Engineering, Taylor's University, Vol. 6, No. 2 (2011) 179 - 190, 2011.

[28] R. M. Lawson, and R. G. Ogden, "Sustainability and Process Benefits of Modular Construction", Proceedings of 18th CIB World Building Congress, TG57 - Industrialization in Construction, May 2010, Salford, UK, CIB Publication 354, p.38-51, 2010.

[29] A. R. B. Zainal Abidin, "Simulation of Industrialized Building System Formation for Housing Construction", MSc Thesis, Faculty of Civil Engineering, University of Technology, Malaysia, 2007.

[30] A. Saggaff, "Industrialized building system - an innovative construction method", MATEC Web of Conferences, 101, 05001 (2017), Sriwijaya International Conference on Engineering, Science and Technology - SICEST 2016, Bangka Island, Indonesia, 2017.

[31] E. M. Generalova, V. P. Generalov and A. A. Kuznetsova, "Modular buildings in modern construction", Procedia Engineering, Elsevier, 153 (2016) 167 - 172, 2016.

[32] M. N. A. Azman, K. A. M. Kamar and M. N. M. Nawi, "Industrialized Building System in Reducing Waste of Construction Industry", Journal of Science Teacher Education - JSTE, Vol. 2, February 2013, p. 96-103, 2013.

[33] A. Mirakhorli and B. Dong, "An Open Source Smart Building Energy Management Platform through VOLTTRON", Proceedings of the 15th IBPSA Conference, International Building Performance Simulation Association, San Francisco, CA, USA, Aug.7-9, 2017, p. 1763$1770,2017$.

[34] S. R. Ermolli, "The Environmental Benefits of the Off-Site Manufacturing", Sustainable Construction, Materials and Practices: Challenge of the Industry for the New Millennium, 12-14 Sep 2007, Lisbon, Portugal, SB07 Proceedings, Published by IOS Press - Delft University Press, The Netherlands, P. 974-981.

[35] K. A. M. Kamar, M. Alshawi and Z. Hamid, "Barriers to Industrialized Building Systems: The 
Case of Malaysia", Proceedings of 9th International Postgraduate Research Conference (IPGRC 2009), The Research Institute for Built and Human Environment $(\mathrm{BuHu})$, The University of Salford, 29th -30th January, 2009, Salford, UK.

[36] K. A. M. Kamar, Z. A. Hamid and M. Alshawi, "The Critical Success Factors (CSFs) to the Implementation of Industrialized Building System (IBS) in Malaysia", Proceedings of 18th CIB World Building Congress, TG57 Industrialization in Construction, May 2010, Salford, UK, CIB Publication 354, p.64-76.

[37] U. Kassim and L. Walid, "Awareness of the Industrialized Building System (IBS) Implementation in Northern Malaysia - A Case Study in Perlis", Procedia Engineering, Elsevier, 53 (2013) 58 - 63, 2013.

[38] Y. Gan, L. Shen, J. Chen, V. W. Y. Tam, Y. Tan and I. M. C. S. Illankoon, "Critical Factors Affecting the Quality of Industrialized Building System Projects in China”, Sustainability (Journal), Molecular Diversity Preservation International - MDPI 2017, 9, 216, p.1-13.

[39] S. Navaratnam, T. Ngo, T. Gunawardena and D. Henderson, "Performance Review of Prefabricated Building Systems and Future Research in Australia”, Buildings (Journal), Molecular Diversity Preservation International MDPI 2019, 9, 38, p.1-13, 2019.

[40] K. A. M. Kamar, M. Alshawi and Z. Hamid, "Industrialized Building System: The Critical Success Factors", Proceedings of 9th International Postgraduate Research Conference (IPGRC 2009), The Research Institute for Built and Human Environment $(\mathrm{BuHu})$, The University of Salford, 29th -30th January, 2009, Salford, UK, p. 485-497.

[41] M. A. O. Mydin, M. N. M. Nawi, M. Y. M. Yunos and N. Utaberta, "Decisive Success Factors in Executing Prefabrication System in Malaysia", Australian Journal of Basic and Applied Sciences, 9(7) April 2015, P. 160-163.

[42] S. S. Kamaruddin, M. F. Mohammad, R. Mahbub and K. Ahmad, "Mechanization and Automation of the IBS Construction Approach: A Malaysian experience", Procedia - Social and Behavioral Sciences, Elsevier, 105 (2013) 106 - 114.
[43] M. F. Musa, M. F. Mohammad, M. R. Yusof and A. Rofizlan, "Industrialized Building System Modular System (IBSMS) Organizational Readiness Framework", Procedia - Social and Behavioral Sciences, Elsevier, 222 (2016) 83 - 92.

[45] M. R. Abdullah and C. Egbu, "The Role of Knowledge Management in Improving the Adoption and Implementation Practices of Industrialized Building System (IBS) in Malaysia”, CIB World Conference 2010, Vol. 1, School of Built Environment, University of Salford.

[45] F. Ismail, N. M. Yusuwan and H. E. A. Baharuddin, "Management Factors for Successful IBS Projects Implementation”, Procedia - Social and Behavioral Sciences, Elsevier, 68 (2012) 99 107.

[46] M. N. M. Nawi, A. Lee, and K. M. Nor, "Barriers to implementation of the industrialized building system (IBS) in Malaysia", The Built and Human Environment Review, Vol.4, p. 22-35, 2011.

[47] M. Qays, K. N. Mustapha, H. Al-Mattarneh and B. S. Mohamed, "The Constraints of Industrialized Building System from Stakeholders' Point of View", IEEE International Conference on Semiconductor Electronics (ICSE2010).

[48] Y. F. Badir, M. R. Abdul Kadir and A. H. Hashim, "Industrialized Building Systems Construction in Malaysia", Journal of Architectural Engineering, Vol. 8, No. 1, ASCE, p. 1-5, 2002.

[49] A. B. A. Rahman and W. Omar, "Issues and Challenges in the Implementation of Industrialized Building Systems Construction in Malaysia", Proceedings of the 6th Asia-Pacific Structural Engineering and Construction Conference (APSEC 2006), 5 - 6 September 2006, Kuala Lumpur, Malaysia, p. C45-C53.

[50] M. Samari, N. Ghodrati and M. W. M. Shafiei, "The Implementation of Industrialized Building System (IBS) in Iran Construction Companies", IOSR Journal of Mechanical and Civil Engineering, Vol. 1, Issue 3, P. 19-24, 2012.

[51] F. M. Andrews, and J. P. Robinson, "Measures of subjective wellbeing", Measures of personality and social psychological attitudes: Measures of social psychological attitudes, Vol. 1, J. P. Robinson, P. R. Shaver, and L. S. Wrightsman, eds., Academic Press, San Diego, 61-114, 1991. 